\title{
Psychological characteristics, stressful life events and deliberate self-harm: findings from the Child \& Adolescent Self-harm in Europe (CASE) Study
}

\author{
Nicola Madge $\cdot$ Keith Hawton · Elaine M. McMahon • Paul Corcoran • \\ Diego De Leo $\cdot$ Erik Jan de Wilde $\cdot$ Sandor Fekete $\cdot$ Kees van Heeringen \\ Mette Ystgaard $\cdot$ Ella Arensman
}

Received: 7 January 2011/ Accepted: 28 July 2011

(C) Springer-Verlag 2011

\begin{abstract}
There is evidence to suggest that both psychological characteristics and stressful life events are contributory factors in deliberate self-harm among young people. These links, and the possibility of a dose-response relationship between self-harm and both psychological health and life events, were investigated in the context of a seven-country school-based study. Over 30,000, mainly 15 and 16 year olds, completed anonymous questionnaires at secondary schools in Belgium, England, Hungary, Ireland, the Netherlands, Norway and Australia. Pupils were asked to report on thoughts and episodes of self-harm, complete scales on depression and anxiety symptoms, impulsivity
\end{abstract}

N. Madge $(\square)$

Centre for Child and Youth Research, Mary Seacole Building,

Brunel University, Uxbridge UB8 3PH, UK

e-mail: Nicola.madge@brunel.ac.uk

K. Hawton

Centre for Suicide Research, University of Oxford, Oxford, UK

E. M. McMahon · P. Corcoran · E. Arensman

National Suicide Research Foundation, Cork, Ireland

D. De Leo

Australian Institute for Suicide Research and Prevention,

Griffith University, Brisbane, Australia

E. J. de Wilde

Netherlands Youth Institute, Utrecht, NL, Netherlands

S. Fekete

Department of Psychiatry, University of Pécs, Pécs, Hungary

K. van Heeringen

Unit of Suicide Research, Ghent University, Ghent, Belgium

M. Ystgaard

Centre for Child \& Adolescent Mental Health, Oslo, Norway and self-esteem and indicate stressful events in their lives. Level and frequency of self-harm was judged according to whether they had thought about harming themselves or reported single or multiple self-harm episodes. Multinomial logistic regression assessed the extent to which psychological characteristics and stressful life events distinguished between adolescents with different self-harm histories. Increased severity of self-harm history was associated with greater depression, anxiety and impulsivity and lower self-esteem and an increased prevalence of all ten life event categories. Female gender, higher impulsivity and experiencing the suicide or self-harm of others, physical or sexual abuse and worries about sexual orientation independently differentiated single-episode self-harmers from adolescents with self-harm thoughts only. Female gender, higher depression, lower self-esteem, experiencing the suicide or self-harm of others, and trouble with the police independently distinguished multiple- from singleepisode self-harmers. The findings reinforce the importance of psychological characteristics and stressful life events in adolescent self-harm but nonetheless suggest that some factors are more likely than others to be implicated.

Keywords Adolescence $\cdot$ Cross-cultural $\cdot$ Self-harm · Depression · Anxiety · Impulsivity · Self-esteem · Stressful life events $\cdot$ Psychological characteristics

\section{Background}

Deliberate self-harm is a significant problem among the young. Although hard to predict, and often appearing 'out of the blue', there is evidence to suggest that mental health problems, impulsivity, self-esteem and stress in young people's lives are contributory factors. We examined 
associations between self-harm and these factors among a large sample of (mainly) 15- and 16-year olds participating in the Child \& Adolescent Self-harm in Europe (CASE) Study and build on earlier findings from this international research [28, 42, 47].

Considerable evidence links psychological characteristics with self-harm thoughts and behaviour in adolescents [14]. These problems may be state-dependent (e.g. depression or anxiety) or trait-dependent (e.g. impulsivity and self-esteem). Associations with depression are particularly widely reported [1, 26, 35], including longitudinal studies [16, 44]. Anxiety symptoms also increase risk of self-harm, particularly when associated with depression $[14,18,41]$.

Impulsivity has been linked with both self-harm episodes and suicidal ideation [10, 21, 25, 26]. Brent and Mann [5] implicate aggressive impulsivity in the interpretation of patterns of suicidal behaviour across generations. Self-esteem (in relation to peers, school, family, sports/ athletics, body image and global self-worth) has also been related to suicidal thoughts and attempts [25, 46].

Self-harm shows strong links with stress factors [20], including difficulties in familial relationships [7, 23, 31], poor relationships with friends and partners [11,23] and perceptions of poor academic performance [30].

In addition, knowing someone who has self-harmed, or made a suicide attempt, contributes to risk [5, 6, 14, 27, 32]. Gay, lesbian and bisexual young people appear at elevated risk [3, 39], especially when facing family difficulties [12]. Child abuse, especially sexual abuse, has repeatedly been associated with self-harming and suicidal behaviour [4, 19, 48], and the link appears direct even though self-esteem [15] or continued adversity over the life-cycle [19] may play a mediating role. Both being bullied [8] and fear of bullying [2] have been linked to an increase in self-harm.

In this paper, we explore links between psychological characteristics, life events and self-harm history among young people within a large international dataset. In particular, we examine the dose-response hypothesis that increasing adversity, in terms of psychological characteristics and life events, is associated with increasing level and frequency of self-harm. Such a relationship between depression and selfharming behaviour has already been suggested for both frequency [13, 19, 22] and severity [35] of episodes.

\section{Methods}

\section{The CASE Study}

This paper draws on data from the CASE Study, an internationally collaborative investigation of self-harm among young people in seven self-selected countries. These comprised six European countries-Belgium, England, Hungary, Ireland, the Netherlands and Norway-and Australia. The study methodology was similar in each participating country and is described in detail elsewhere [28]. School-based surveys were conducted with a total of 30,477 14-17-year-old adolescents, the majority being 15 or 16 years, who consented to provide anonymous selfreport data on self-harm behaviour (e.g. timing, frequency of episodes, methods used, motives, help-seeking before and after the episode, hospitalisation, serious thoughts about self-harm), negative life events, lifestyle and psychological characteristics including symptoms of anxiety, depression, self-esteem and impulsivity. Schools were selected to be locally and nationally representative, and response rates ranged from 81 to 96 per cent in individual countries.

\section{Measures}

\section{Self-harm}

To ensure international comparability, strict criteria were adopted to assess self-harm taking account of whether young people said they had harmed themselves as well as descriptions of the last episode. The criterion for self-harm depended on a report of at least one of the following acts deliberately undertaken with non-fatal outcome:

- Initiated behaviour (e.g. self-cutting, jumping from a height), which they intended to cause self-harm;

- Ingested a substance in excess of the prescribed or generally recognised therapeutic dose;

- Ingested a recreational or illicit drug that was an act that the person regarded as self-harm;

- Ingested a non-ingestible substance or object.

Judgements of self-harm were in no way dependent on motives other than that the act in question appeared deliberate. It was not possible to draw a clear distinction between suicidal and non-suicidal self-harm from the survey data. Further information on the assessment of selfharm is provided elsewhere [28].

Study participants were divided into four groups: "no self-harm' comprising all those who reported neither selfharm behaviour meeting the study criteria nor thoughts of self-harm, in the past year; 'self-harm thoughts only' with self-harm thoughts but no episode of self-harm in the past year; 'single self-harm episode' with one episode in the previous year; and 'multiple self-harm episodes' with an episode in the previous year as well as at least one other earlier episode. 


\section{Psychological characteristics}

Four psychological scales were included. Anxiety and depression symptoms were measured by the HADS scale [49], which includes two 7-item subscales for anxiety and depression, each scored between 0 and 21, where higher scores indicated higher levels of anxiety and depression. Self-esteem was measured using an eight-item abbreviated version of the Robson [40] self-concept scale, with possible scores between 8 and 32, and higher scores indicating more positive self-esteem. A six-item impulsivity scale [37] led to scores between 6 and 24 with higher scores indicating greater impulsivity. Raw scores on these measures are used in the analysis.

\section{Life events}

The questionnaire included 20 questions relating to negative life events in the past 12 months and/or more than a year ago. For the purposes of analysis, these were reduced to ten categories of lifetime experience. These were: 'Difficulties with friends and peers'; 'Problems with or between parents'; 'Serious illness of family or friend'; 'Physical or sexual abuse'; 'Suicide or self-harm of family or friend'; 'Death of someone close'; 'Worries about sexual orientation'; 'Trouble with police'; 'Bullied'; and 'Problems with schoolwork'.

\section{Data analysis}

All analyses were undertaken for the sample as a whole, and some analyses were carried out by gender and by country. Chi-square tests were used to assess the statistical significance of associations between pairs of categorical variables such as self-harm history and gender. The strength of these associations was measured by the Phi statistic. In line with previous recommendations [9], associations were considered very weak if Phi $<0.10$, weak if $<0.30$, moderate if $<0.50$ and strong if $0.50+$.

Cronbach's alpha was used to assess the internal consistency of the scales employed to measure the psychological characteristics. These measures (depression, anxiety, impulsivity, self-esteem) and the total number of stressful life event categories experienced followed a normal distribution and were summarised by the mean and standard deviation. Pearson's correlation coefficient, $r$, was used to assess the strength of the linear association between the psychological measures, and the coefficient of determination, $r^{2}$, was used to measure the information in one measure that could be explained statistically by another.

Between-group comparisons of the psychological measures and the number of reported stressful life event categories were carried out using analysis of variance. The effect size was measured using partial $\eta^{2}$, and, following established guidelines [9], the effect size was considered very small if partial $\eta^{2}<0.01$, small if $<0.06$, medium if $<0.14$ and large if $0.14+$. Post hoc pairwise comparisons were based on Tukey's honestly significant difference.

Multinomial logistic regression was used to assess the associations between gender, age, country, psychological characteristics and reported stressful life event categories and self-harm history in the past year with a view to identifying which factors distinguished between adolescents reporting no self-harm, self-harm thoughts only, a single self-harm episode and multiple self-harm episodes. The dependent variable comparison group was the group with no self-harm in the past year. Gender, age and country were entered into the regression model as the first block of independent variables. A forward stepwise approach was adopted for the second block of independent variables which contained each reported stressful life event category, the total number of reported life event categories and the psychological characteristics. Associations were reported as odds ratios with $95 \%$ confidence intervals. Nagelkerke's $r^{2}$ was used as the estimate of the proportion of variance explained by the derived regression model. Wald tests were carried out to identify the factors distinguishing adolescents with a single self-harm episode from those with self-harm thoughts only and factors distinguishing between multiple- and single-episode selfharmers.

Further multinomial logistic regression models were estimated in which interaction terms (by country, by gender and between the psychological measures) were considered as a third block of factors for entry into the multivariate regression model described previously. Change in Nagelkerke's $r^{2}$ was used to quantify the extent to which interaction terms further distinguished between the four self-harm history groups.

\section{Results}

Self-harm history in past year

Overall, $79.6 \%$ of the young people said they had not experienced thoughts of self-harm in the past year, $14.6 \%$ said they had thought about harming themselves but had not done so, $2.6 \%$ reported single self-harm episodes in the past year meeting the study criteria, and a further $3.2 \%$ reported multiple episodes. Females were at least twice as likely as males to report having thoughts of self-harm and both single- and multiple-episodes of self-harm (Table 1). While statistically significant at $P<0.001$, the strength of the association between self-harm history and gender was weak $(\phi=0.22)$. 
Table 1 Psychological characteristics, reported stressful life events and self-harm history in past year by gender

\begin{tabular}{|c|c|c|c|c|c|c|c|c|c|}
\hline & & \multicolumn{2}{|l|}{ All } & \multicolumn{2}{|l|}{ Male } & \multicolumn{2}{|c|}{ Female } & \multirow[t]{2}{*}{$P$-value } & \multirow{2}{*}{$\begin{array}{l}\text { Partial } \\
\eta^{2}\end{array}$} \\
\hline & & Mean & (SD) & Mean & (SD) & Mean & (SD) & & \\
\hline \multirow{4}{*}{$\begin{array}{l}\text { Psychological } \\
\text { characteristics }\end{array}$} & Depression & 4.4 & $(3.3)$ & 4.4 & (3.3) & 4.3 & (3.3) & Not sig. & $<0.01$ \\
\hline & Anxiety & 6.9 & $(4.0)$ & 6.1 & $(3.8)$ & 7.8 & $(4.1)$ & $<0.001$ & 0.04 \\
\hline & Impulsivity & 13.9 & $(2.9)$ & 13.8 & $(2.9)$ & 14.1 & (2.9) & $<0.001$ & $<0.01$ \\
\hline & Self-esteem & 22.4 & $(3.9)$ & 23.1 & $(3.8)$ & 21.7 & $(4.0)$ & $<0.001$ & 0.03 \\
\hline \multirow[t]{12}{*}{ Life event category } & Number of life event categories & 3.6 & $(2.1)$ & 3.3 & $(2.0)$ & 3.9 & $(2.1)$ & $<0.001$ & 0.02 \\
\hline & & $n$ & $(\%)$ & $n$ & $(\%)$ & $n$ & $(\%)$ & $P$-value & $\phi$ \\
\hline & Death of someone close & 18203 & $(59.7 \%)$ & 8776 & $(56.3 \%)$ & 9427 & $(63.4 \%)$ & $<0.001$ & 0.07 \\
\hline & Problems with or between parents & 16895 & $(55.4 \%)$ & 7844 & $(50.2 \%)$ & 9051 & $(60.8 \%)$ & $<0.001$ & 0.11 \\
\hline & Serious illness of family or friend & 16753 & $(55.0 \%)$ & 8232 & $(52.8 \%)$ & 8521 & $(57.3 \%)$ & $<0.001$ & 0.05 \\
\hline & Difficulties with friends and peers & 16284 & $(53.3 \%)$ & 6999 & $(44.8 \%)$ & 9285 & $(62.3 \%)$ & $<0.001$ & 0.18 \\
\hline & Problems with schoolwork & 14414 & $(47.5 \%)$ & 6943 & $(44.7 \%)$ & 7471 & $(50.4 \%)$ & $<0.001$ & 0.06 \\
\hline & Suicide/self-harm of others & 9279 & $(30.4 \%)$ & 3369 & $(21.6 \%)$ & 5910 & $(39.7 \%)$ & $<0.001$ & 0.20 \\
\hline & Bullied & 6339 & $(20.9 \%)$ & 2955 & $(19.0 \%)$ & 3384 & $(22.9 \%)$ & $<0.001$ & 0.05 \\
\hline & Trouble with the police & 5318 & $(17.5 \%)$ & 3751 & $(24.2 \%)$ & 1567 & $(10.6 \%)$ & $<0.001$ & 0.18 \\
\hline & Physical or sexual abuse & 3164 & $(10.4 \%)$ & 1258 & $(8.0 \%)$ & 1906 & $(12.8 \%)$ & $<0.001$ & 0.08 \\
\hline & Worries about sexual orientation & 1737 & $(5.7 \%)$ & 746 & $(4.8 \%)$ & 991 & $(6.7 \%)$ & $<0.001$ & 0.04 \\
\hline \multirow[t]{4}{*}{ Self-harm history } & No self-harm & 23038 & $(79.6 \%)$ & 13020 & $(88.1 \%)$ & 10018 & $(70.7 \%)$ & $<0.001$ & 0.22 \\
\hline & Self-harm thoughts only & 4237 & $(14.6 \%)$ & 1365 & $(9.2 \%)$ & 2872 & $(20.3 \%)$ & & \\
\hline & Single self-harm episode & 752 & $(2.6 \%)$ & 179 & $(1.2 \%)$ & 573 & $(4.0 \%)$ & & \\
\hline & Multiple self-harm episodes & 912 & $(3.2 \%)$ & 212 & $(1.4 \%)$ & 700 & $(4.9 \%)$ & & \\
\hline
\end{tabular}

Effect size measured by partial $\eta^{2}$ was very small if $<0.01$, small if $<0.06$, medium if $<0.14$ or large if $0.14+$

Association measured by $\phi$ was very weak if $<0.10$, weak if $<0.30$, moderate if $<0.50$ and strong if $0.50+$

Some differences emerged between countries [see also 28]. The Netherlands, in particular, stood out as having low rates of both thoughts and episodes of self-harm among both males and females. Hungary also showed an interesting pattern in that, compared with other countries, both males and females were less likely to report no thoughts or episodes of self-harm in the previous year, but more likely to report self-harm thoughts only.

\section{Psychological characteristics}

The psychological measures had satisfactory levels of internal consistency (Cronbach's alpha: 0.69 for depression, 0.84 for anxiety, 0.75 for impulsivity and 0.90 for self-esteem). All intercorrelations between depression, anxiety, impulsivity and self-esteem were statistically significant at $P<0.001$. There was only one strong correlation that was between depression and anxiety (Pearson's correlation coefficient, $\left.r=0.52 ; r^{2}=0.27\right)$. Depression and anxiety were equally negatively correlated with selfesteem $\left(r=-0.45 ; r^{2}=0.20\right)$. Other correlations were weak, ranging in magnitude from 0.13 to 0.24 $\left(r^{2}=0.02-0.06\right)$, indicating that there was limited overlap between the psychological measures.
There was no gender difference in relation to depression (Table 1). Female scores were generally higher than male scores on anxiety and impulsivity and lower on selfesteem. However, the effect size was small for anxiety and self-esteem and very small for impulsivity.

Depression, anxiety, impulsivity and self-esteem differed by country $(P<0.001$ in each case $)$ with partial $\eta^{2}$ ranging from 0.03 to 0.04 and indicating a small effect of country on psychological measures. Mean scores ranged from 3.6 in Ireland to 5.7 in Hungary for depression, between 6.0 in Norway and 8.0 in England for anxiety, between 13.2 in Belgium and 14.8 in Hungary for impulsivity, and between 21.3 in Hungary and 23.3 in Norway for self-esteem. Overall Hungarian adolescents stood out as displaying the highest levels of psychological difficulties, while Norwegian adolescents showed the lowest levels.

\section{Stressful life event categories}

The reported stressful life event categories varied widely in prevalence from $59.7 \%$ for the death of someone close down to $5.7 \%$ for worries about sexual orientation. On average, 3.6 categories were reported with female students 
Table 2 Psychological characteristics by self-harm history in past year

\begin{tabular}{lcccccc}
\hline & $\begin{array}{l}\text { No self-harm } \\
\text { Mean (SD) }\end{array}$ & $\begin{array}{l}\text { Self-harm thoughts only } \\
\text { Mean (SD) }\end{array}$ & $\begin{array}{l}\text { Single self-harm episode } \\
\text { Mean (SD) }\end{array}$ & $\begin{array}{l}\text { Multiple self-harm episodes } \\
\text { Mean (SD) }\end{array}$ & $P$-value & Partial $\eta^{2}$ \\
\hline Depression & $3.9(3.0)$ & $5.6(3.3)$ & $5.9(3.6)$ & $7.4(4.2)$ & $<0.001$ & 0.08 \\
Anxiety & $6.1(3.7)$ & $9.5(3.8)$ & $9.9(4.1)$ & $11.4(4.2)$ & $<0.001$ & 0.11 \\
Impulsivity & $13.6(2.8)$ & $14.8(3.0)$ & $15.3(3.1)$ & $15.9(3.2)$ & $<0.001$ & 0.04 \\
Self-esteem & $23.1(3.7)$ & $20.3(3.7)$ & $20.1(4.0)$ & $18.4(4.2)$ & $<0.001$ & 0.09 \\
\hline
\end{tabular}

Effect size measured by partial $\eta^{2}$ was very small if $<0.01$, small if $<0.06$, medium if $<0.14$ or large if $0.14+$

All pairwise comparisons among the four self-harm history groups differed significantly at $P<0.001$ with the exception of the comparison between the self-harm thoughts only group and the single self-harm episode group in relation to depression $(P=0.131)$, anxiety $(P=0.012)$ and self-esteem $(P=0.477)$

reporting a higher number than male students. The prevalence of each life event category differed by gender at $P<0.001$. For all but one stressful life event category (trouble with the police), the prevalence was higher among female students. However, the strength of the association between prevalence of stressful life events and gender was weak or very weak (range of Phi statistic $=0.04-0.20$ ). The prevalence of each stressful life event category varied by country (all at $P<0.001$ ), but the strength of all associations was weak or very weak.

Psychological characteristics and self-harm history in past year

There were highly statistically significant differences $(P<0.001)$ between the self-harm history groups on each of the psychological characteristics (Table 2). The effect size was small in relation to impulsivity and medium in relation to depression, anxiety and self-esteem. There was evidence of a dose-response or graded relationship whereby the more severe the self-harm history, the higher the levels of depression, anxiety and impulsivity and the lower the level of self-esteem. All pairwise comparisons among the four self-harm history groups differed significantly at $P<0.001$ with three exceptions. The self-harm thoughts only group and the single self-harm episode group did not differ significantly in relation to depression $(P=0.131)$ and self-esteem $(P=0.477)$ and only differed marginally in relation to anxiety $(P=0.012)$.

Figure 1 illustrates the consistency of the doseresponse association between the four psychological characteristics and self-harm history when examined for each of the seven countries. The stepped increase in depression, anxiety and impulsivity and decrease in selfesteem was evident with increasing self-harm history. However, in most countries, there was limited or no difference in level of depression, anxiety and self-esteem between the adolescents who only thought of self-harming but did not act and those who engaged in a single self-harm episode. There was no evidence of gender modifying the association between the psychological measures and self-harm history.

Stressful life event categories and self-harm history in past year

There were highly statistically significant $(P<0.001)$ associations between the reporting of each life event category and self-harm history in the past year (Table 3 ). In every case, the prevalence increased across the groups with increasing selfharm history. The life event category most strongly related to self-harm history was experiencing the suicide or self-harm of others followed by physical or sexual abuse, difficulties with friends or peers and problems with or between parents. There was also evidence of a strong dose-response relationship as the average number of event categories reported varied from 3.1 for adolescents with no self-harm in the past year to 4.8 for those with self-harm thoughts only to 5.5 for those with a single self-harm episode and 6.2 for multiple self-harmers. Figure 1 illustrates this dose-response association.

Gender modified the association between three of the life event categories (death of someone close $(P=0.001)$, bullied $(P=0.013)$ and worries about sexual orientation $(P<0.001))$ and self-harm history. Country modified the association between six of the life event categories (difficulties with friends and peers $(P=0.002)$, problems with schoolwork $(P=0.025)$, suicide/self-harm of others $(P<0.001)$, bullied $(P<0.001)$, physical or sexual abuse $(P<0.001)$ and worries about sexual orientation $(P<0.001))$ and self-harm history. However, none of these interaction effects explained more than an additional $0.2 \%$ of the variation in self-harm history.

Independent associations between gender, psychological characteristics and stressful life event categories and self-harm history in past year

Table 4 details the results of the multivariate multinomial logistic regression analysis carried out to identify the factors independently distinguishing between adolescents in the four self-harm history groups. The derived regression 

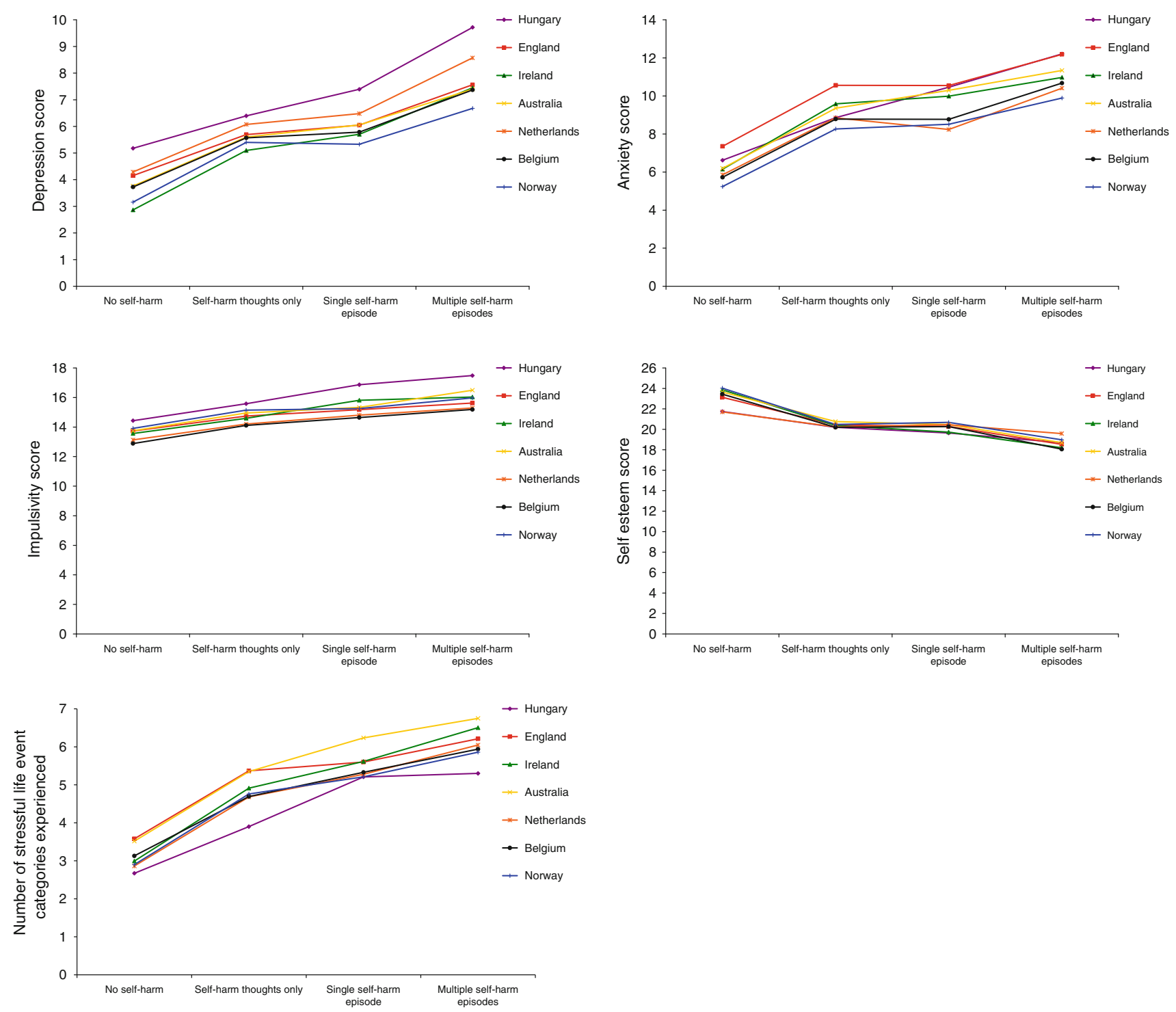

Fig. 1 Mean depression, anxiety, impulsivity, self-esteem scores and mean number of stressful life event categories by self-harm history in past year for each country

model explained $37.7 \%$ of the variation in self-harm history. Some interactions between the psychological measures with gender and with country reached statistical significance but they contributed very little $(0.2-0.8 \%)$ to explaining variation in self-harm history.

Female gender strongly distinguished self-harm ideators and single and multiple self-harmers from those with no self-harm history. Female gender also distinguished adolescents with a single self-harm episode from those with self-harm thoughts only $(P<0.001)$ but did not distinguish between single and multiple self-harmers $(P=0.523)$.

Each of the four psychological characteristics independently contributed to distinguishing between the self-harm groups. Only impulsivity distinguished single-episode self-harmers from self-harm ideators $(P=0.005)$, whereas self-esteem
$(P<0.001)$ and depression $(P=0.002)$ differentiated multiple self-harmers from those with a single episode.

All but one (death of someone close) of the ten stressful life event categories was independently associated with self-harm history (Table 4). Experience of the suicide/self-harm of others strongly distinguished adolescents with a single selfharm episode from those with self-harm thoughts only $(P<0.001)$ and also distinguished multiple- from singleepisode self-harmers $(P=0.015)$. Physical or sexual abuse $(P<0.001)$ and to a lesser extent worries about sexual orientation $(P=0.017)$ differentiated between adolescents with a single self-harm episode from those with self-harm thoughts only, while trouble with the police was associated with multiple self-harmers more than it was with single-episode selfharmers $(P=0.019)$. 
Table 3 Prevalence of stressful life event categories by self-harm history in past year

\begin{tabular}{|c|c|c|c|c|c|c|c|c|c|}
\hline & \multicolumn{2}{|c|}{ No self-harm } & \multicolumn{2}{|c|}{ Self-harm thoughts only } & \multicolumn{2}{|c|}{$\begin{array}{l}\text { Single self-harm } \\
\text { episode }\end{array}$} & \multicolumn{2}{|c|}{$\begin{array}{l}\text { Multiple self-harm } \\
\text { episodes }\end{array}$} & \multirow[t]{2}{*}{$\phi^{\mathrm{a}}$} \\
\hline & $n$ & $(\%)$ & $n$ & $(\%)$ & $n$ & $(\%)$ & $n$ & $(\%)$ & \\
\hline Death of someone close & 13249 & $(57.5 \%)$ & 2778 & $(65.6 \%)$ & 519 & $(69.7 \%)$ & 677 & $(74.2 \%)$ & 0.09 \\
\hline Problems with or between parents & 11263 & $(49.0 \%)$ & 3179 & $(75.2 \%)$ & 623 & $(83.2 \%)$ & 792 & $(87.3 \%)$ & 0.24 \\
\hline Serious illness of family or friend & 11716 & $(51.0 \%)$ & 2838 & $(67.1 \%)$ & 520 & $(69.8 \%)$ & 685 & $(75.5 \%)$ & 0.15 \\
\hline Difficulties with friends and peers & 10739 & $(46.7 \%)$ & 3180 & $(75.1 \%)$ & 599 & $(79.7 \%)$ & 776 & $(85.2 \%)$ & 0.25 \\
\hline Problems with schoolwork & 9573 & $(41.8 \%)$ & 2716 & $(64.7 \%)$ & 531 & $(71.5 \%)$ & 694 & $(76.5 \%)$ & 0.21 \\
\hline Suicide/self-harm of others & 5281 & $(22.9 \%)$ & 2108 & $(49.8 \%)$ & 525 & $(69.9 \%)$ & 722 & $(79.3 \%)$ & 0.32 \\
\hline Bullied & 4059 & $(17.8 \%)$ & 1267 & $(30.2 \%)$ & 263 & $(35.3 \%)$ & 394 & $(43.9 \%)$ & 0.16 \\
\hline Trouble with the police & 3546 & $(15.5 \%)$ & 831 & $(19.7 \%)$ & 191 & $(25.7 \%)$ & 314 & $(34.6 \%)$ & 0.10 \\
\hline Physical or sexual abuse & 1474 & $(6.4 \%)$ & 808 & $(19.1 \%)$ & 233 & $(31.0 \%)$ & 352 & $(38.7 \%)$ & 0.26 \\
\hline Worries about sexual orientation & 882 & $(3.8 \%)$ & 428 & $(10.2 \%)$ & 120 & $(16.0 \%)$ & 182 & $(20.2 \%)$ & 0.17 \\
\hline Number of life event categories ${ }^{b}$ & 3.1 & (1.9) & 4.8 & $(1.9)$ & 5.5 & $(1.9)$ & 6.2 & $(1.8)$ & 0.16 \\
\hline
\end{tabular}

All associations statistically significant at $P<0.001$

${ }^{a}$ Association measured by Phi was very weak if $<0.10$, weak if $<0.30$, moderate if $<0.50$ and strong if $0.50+$

b Mean (standard deviation) reported with partial $\eta^{2}$ as the effect size measure. Effect size measured by partial $\eta^{2}$ was very small if $<0.01$, small if $<0.06$, medium if $<0.14$ or large if $0.14+$. All post hoc pairwise comparisons among the four self-harm history groups were statistically significant at $P<0.001$

\section{Discussion}

An earlier paper from the CASE Study [28] addressed socio-demographic and self-harm characteristics for the same school-based sample of adolescents. Here, we have focused in more depth on psychological characteristics and stressful life events. In particular, we have reported evidence for a dose-response relationship whereby increased severity of self-harm history was, in general, associated with higher levels of depression, anxiety and impulsivity and lower levels of self-esteem, as well as stressful life events in more areas of young people's lives. These patterns were consistent across gender and country.

A particularly interesting finding is that thoughts of selfharm were not always distinguishable from a single selfharm episode in terms of association with psychological characteristics and stressful life events. It emerged, however, that impulsivity and experiencing the suicide or selfharm of others, physical or sexual abuse and worries about sexual orientation were the only factors that independently differentiated single-episode self-harmers from adolescents with self-harm thoughts only. That few factors distinguished between ideators and self-harmers is important for future research and for prevention. The findings are also in line with a recently presented model of suicidal behaviour which maps the relationship between background factors and trigger events, and the development of suicidal ideation/intent through to suicidal behaviour [34].

The strong associations between psychological characteristics and self-harm on the one hand and stressful life events and self-harm on the other raise the question of the associations between psychological health, stressful life events and self-harm, and the relative significance of each set of factors. Multivariate models were constructed to investigate this study objective. Despite some specific differences, the effects of psychological characteristics and stressful life events remained significant within the population overall and continued to remain significant when gender and country were considered separately. Our findings of independent associations between four psychological characteristics and nine life event categories with self-harm history support the conclusion of a systematic review showing that a wide range of factors is linked with self-harm behaviour [14]. It also confirms the heterogeneity of young self-harmers among community samples, with differing frequencies of self-harm and varying levels of associated difficulties [43].

In any event, it would seem unlikely that a single chain of events links life events, psychological health and deliberate self-harm. On the one hand, it is clear that stress can precede mental health difficulties [36, 38]. On the other, however, it is apparent that depression can trigger psychosocial difficulties through poor interpersonal functioning and impaired relationships [42] and that self-harm or suicidal behaviour may follow life stress in the absence of depression and hopelessness [2, 29]. Furthermore, selfharm itself may act as a trigger for depression [17].

Some differences between countries were found. There was, for instance, some variation in levels of self-harm behaviour as well as differences in psychological 


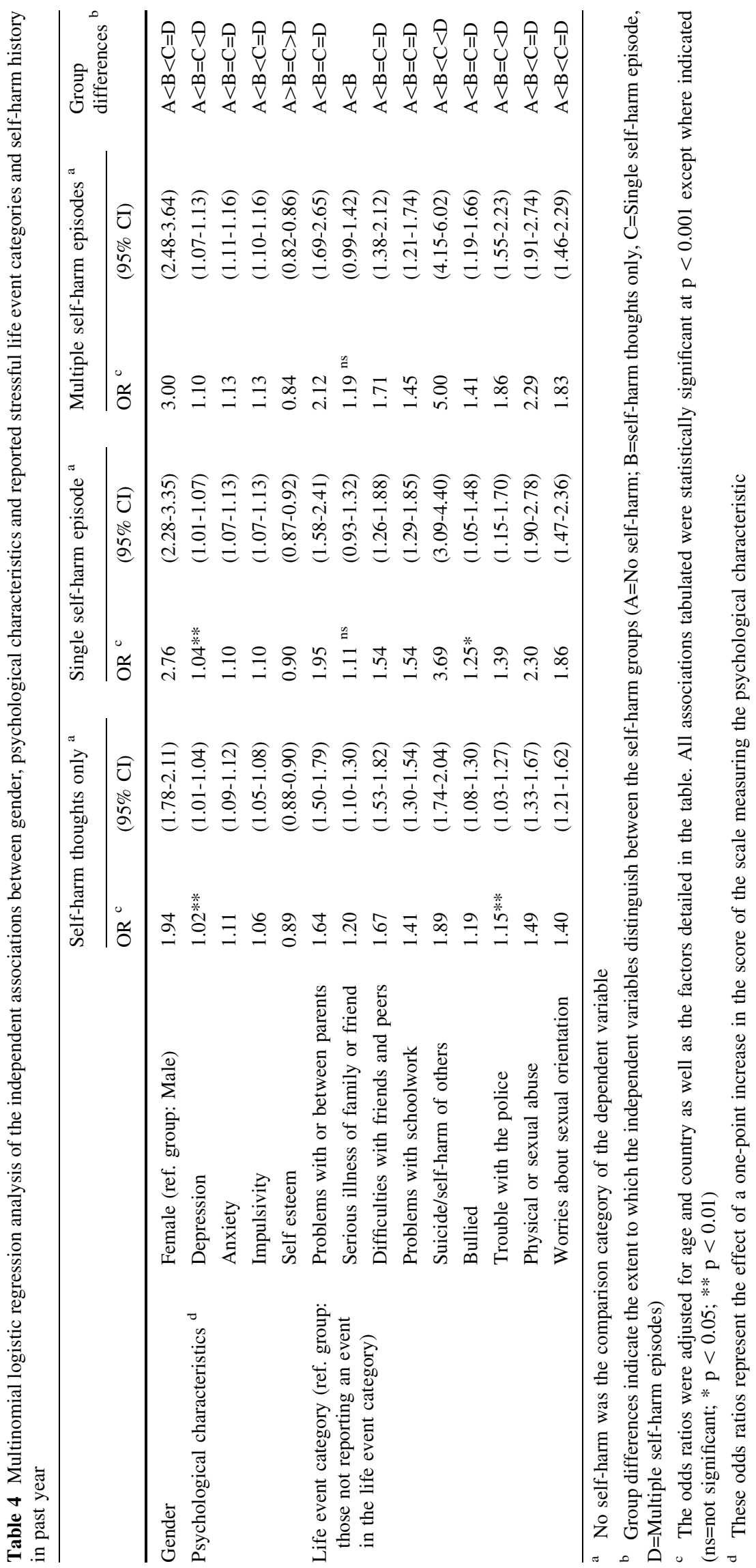


characteristics: in this latter respect, Hungarian young people reported the highest levels of difficulty, while Norwegians reported the lowest. Despite these differences, however, the dose-response relationships between psychological characteristics and history of self-harm, and between stressful life events and self-harm, were maintained in all countries. This suggests the universality of the findings.

These findings underline the complexity involved in specifying the precursors of self-harm among the young. Although both psychological characteristics and negative life events are associated with increased levels of self-harm within the population of young people as a whole, varying constellations of interrelated factors contribute to individual risk. Recent research on identifying subgroups of young self-harmers [43] appears promising, and it is suggested that knowledge on the aetiology of deliberate self-harm may develop best in the context of further large-scale longitudinal research that can look at distinct groups of young self-harmers over time.

The strengths of this study are its large representative school-based sample, the clear definition of self-harm and the standardised methodology used across seven countries. Whilst we relied on retrospective self-reported information, and thus were unable to look conclusively at temporal associations, most of our findings have relied on episodes of self-harm reported for the previous year, rather than over the lifetime, to increase accuracy. The cross-sectional nature of the study, nonetheless, implies that the nature of the relationships between self-harm, psychological characteristics and life events that we have identified cannot be assumed to be causal. Additionally, there may have been other potentially relevant factors, such as substance or alcohol abuse, that we do not consider. A further limitation of the study is that we cannot guarantee the representative nature of all national samples despite attempts to ensure this was the case. Treatment of life events presents another possible methodological difficulty in that categories of stressful events cannot be equated or added. Nonetheless, the fact that all but one stressful life event category showed statistically significant associations with a history of selfharm increases our confidence in the validity of these measures. Further research exploring the impact of different life events, and taking account of severity, occurrence and timing, could throw more light on this issue.

In clinical terms, the evidence for a dose-response relationship linking both psychological characteristics and negative life events with the frequency of self-harm underlines the need for positive mental health promotion and early identification of adolescents at risk of self-harm $[24,33]$. Similarities found between adolescents who have merely thought about harming themselves, and those reporting a single episode, suggest the salience of taking intentions as well as behaviour into account.

\section{Conclusion}

There is no single pattern of self-harm among young people, but both psychological characteristics and stressful life events substantially increase risk. Those developing prevention and intervention programmes must remain 'open minded' to patient characteristics and not neglect either those who have only thought of harming themselves or, despite current practice [45], those who do not have evident signs of depression or mental illness.

Acknowledgments The authors thank the European Commission Daphne Programme for providing funding for the coordination of this research, as well as the Community Fund in England, the Irish National Suicide Review Group, the National Office for Suicide Prevention and the Ireland Funds, QLD-Health and the Commonwealth of Australia, Department of Health and Ageing, and the Norwegian Foundation for Health and Rehabilitation, for funding national studies. We also acknowledge colleagues in the national centres who contributed to the work undertaken, including Udo Reulbach and Tony Fitzgerald for statistical advice, and pupils and schools that participated in the study.

Conflict of interest The authors declare that they have no conflict of interest.

\section{References}

1. Andrews JA, Lewinsohn PM (1992) Suicidal attempts among older adolescents: prevalence and co-occurrence with psychiatric disorders. J Am Acad Child Adolesc Psychiatry 31:655-662

2. Baldry AC, Winkel WF (2003) Direct and vicarious victimization at school and at home as risk factors for suicidal cognition among Italian adolescents. J Adolesc 26:703-716

3. Bearman PS, Moody J (2004) Adolescent suicidality. Am J Pub Health 94:89-95

4. Bensley LS, Van Eenwyk J, Spieker SJ, Schoder MN (1999) Selfreported abuse history and adolescent problem behaviours. I: antisocial and suicidal behaviours. J Adolesc Health 24:163-172

5. Brent D, Mann JJ (2006) Familial pathways to suicidal behavior. N Engl J Med 355:2719-2721

6. Bridge J, Goldstein T, Brent D (2006) Adolescent suicide and suicidal behaviour. J Child Psychol Psychiatry 47:372-394

7. Byrne S, Morgan S, Fitzpatrick C, Boylan C, Crowley S, Gahan H, Howley J, Staunton D, Guerin S (2008) Deliberate self-harm in children and adolescents: a qualitative study exploring the needs of parents and carers. Clin Child Psychol Psychiatry 13:493-504

8. Coggan C, Bennett S, Hooper R, Dickinson P (2003) Association between bullying and mental health status in New Zealand adolescents. Int J Mental Health Promotion 5:16-22

9. Cohen J (1988) Statistical power analysis for the behavioural sciences. Erlbaum, Hillsdale, NJ

10. Conner KR, Meldrum S, Wieczorek WF, Duberstein PR, Welte JW (2004) The association of irritability and impulsivity with suicidal ideation among 15- to 20-year-old males. Suicide Life Threat Behav 34:363-373

11. Dimmock M, Grieves S, Place M (2008) Young people who cut themselves - a growing challenge for educational settings. Br J Spec Edu 35:42-48 
12. Eisenberg ME, Resnick MD (2006) Suicidality among gay, lesbian and bisexual youth: the role of protective factors. J Adolesc Health 39:662-668

13. Esposito C, Spirito A, Boergers J, Donaldson D (2003) Affective, behavioural, and cognitive functioning in adolescents with multiple suicide attempts. Suicide Life Threat Behav 33:389-399

14. Evans E, Hawton K, Rodham K (2004) Factors associated with suicidal phenomena in adolescents: a systematic review of population-based studies. Clin Psychol Rev 24:957-979

15. Evans E, Hawton K, Rodham K (2005) Suicidal phenomena and abuse in adolescents: a review of epidemiological studies. Child Abuse Negl 29:45-58

16. Fergusson DM, Horwood LJ, Ridder EM, Beautrais AL (2005) Suicidal behaviour in adolescence and subsequent mental health outcomes in young adulthood. Psychol Med 35:983-993

17. Flisher AJ (1999) Annotation: mood disorder in suicidal children and adolescents: recent developments. J Child Psychol Psychiatry 40:315-324

18. Foley D, Goldston D, Costello E, Angold A (2006) Proximal psychiatric risk factors for suicidality in youth. Arch Gen Psychiatry 63:1017-1024

19. Harrington R, Pickles A, Aglan A, Harrington V, Burroughs H, Kerfoot M (2006) Early adult outcomes of adolescents who deliberately poisoned themselves. J Am Acad Child Adolesc Psychiatry 45:337-345

20. Hawton K, Harriss L (2008) Deliberate self-harm by under-15year-olds: characteristics, trends and outcome. J Child Psychol Psychiatry 49:441-448

21. Hawton K, Cole D, O'Grady J, Osborn M (1982) Motivational aspects of deliberate self-poisoning in adolescents. Br J Psychiatry 141:286-291

22. Hawton K, Kingsbury S, Steinhardt K, James A, Fagg J (1999) Repetition of deliberate self-harm by adolescents: the role of psychological factors. J Adolesc 22:369-378

23. Hawton K, Hall S, Simkin S, Bale L, Bond A, Codd S, Stewart A (2003) Deliberate self-harm in adolescents: a study of characteristics and trends in Oxford, 1990-2000. J Child Psychol Psychiatry 44:1191-1198

24. Horowitz LM, Ballard ED (2009) Suicide screening in schools, primary care and emergency departments. Curr Opin Pediatr $21: 620-627$

25. Hull-Blanks EA, Kerr BA, Robinson Kurpius SE (2004) Risk factors of suicidal ideations and attempts in talented, at-risk girls. Suicide Life Threat Behav 34:267-276

26. Kerfoot M, Dyer E, Harrington V, Woodham A, Harrington R (1996) Correlates and short-term course of self-poisoning in adolescents. Br J Psychiatry 168:38-42

27. Lieb R, Bronisch T, Hofler M et al (2005) Maternal suicidality and risk of suicidality in offspring: findings from a community study. Am J Psychiatry 162:1665-1671

28. Madge N, Hewitt A, Hawton K, de Wilde EJ, Corcoran P, Fekete S, van Heeringen K, De Leo D, Ystgaard M (2008) Deliberate self-harm within an international community sample of young people: findings from the Child \& Adolescent Self-harm in Europe (CASE) Study. J Child Psychol Psychiatry 49:667-677

29. Martin G, Bergen HA, Richardson AS, Roeger L, Allison S (2004) Sexual abuse and suicidality: gender differences in a large community sample of adolescents. Child Abuse Negl 28:491-503

30. Martin G, Richardson AS, Bergen HA, Roeger L, Allison S (2005) Perceived academic performance, self-esteem and locus of control as indicators of need for assessment of adolescent suicide risk: implications for teachers. J Adolesc 28:75-87
31. McDonald G, O'Brien L, Jackson D (2007) Guilt and shame: experiences of parents of self-harming adolescents. J Child Health Care 11:298-310

32. Melhem NM, Day N, Shear MK, Day R, Reynolds CF, Brent D (2004) Traumatic grief among adolescents exposed to a peer's suicide. Am J Psychiatry 161:1411-1416

33. Naylor PB, Cowie HA, Walters SJ, Talamelli L, Dawkins J (2009) Impact of a mental health teaching programme on adolescents. Br J Psychiatry 194:365-370

34. O'Connor RC (2011) Toward an integrated motivational-volitional model of suicidal behaviour. In: O'Connor RC, Platt S, Gordon J (eds) International handbook of suicide prevention: research, policy and practice, 1st edn. John Wiley \& Sons Ltd., London, pp 181-198

35. Olfson M, Gameroff MJ, Marcus SC, Greenberg T, Shaffer D (2005) Emergency treatment of young people following deliberate self-harm. Arch Gen Psychiatry 62:1122-1128

36. Pelkonen M, Marttunen M, Kaprio J, Huurre T, Aro H (2008) Adolescent risk factors for episodic and persistent depression in adulthood. A 16-year prospective follow-up study of adolescents. J Affect Disord 106:123-131

37. Plutchik R, Van Praag HM (1986) The measurement of suicidality, aggressivity and impulsivity. Clin Neuropharmacol 9:380-382

38. Reinherz H, Tanner J, Berger S et al (2006) Adolescent suicidal ideation as predictive of psychopathology, suicidal behavior, and compromised functioning at age 30 . Am J Psychiatry 163:1126-1232

39. Remafedi G, French S, Story M, Resnick M, Blum R (1998) The relationship between suicide risk and sexual orientation: results of a population-based study. Am J Public Health 88:57-60

40. Robson P (1989) Development of a new self-report questionnaire to measure self-esteem. Psychol Med 19:513-518

41. Ross S, Heath NL (2003) Two models of adolescent self-mutilation. Suicide Life Threat Behav 33:277-287

42. Rossow I, Ystgaard M, Hawton K, Madge N, van Heeringen K, de Wilde EJ, De Leo D, Fekete S, Morey C (2007) Cross-national comparisons of the association between alcohol consumption and deliberate self-harm in adolescents. Suicide Life Threat Behav 37:605-615

43. Stanford S, Jones MP (2009) Psychological subtyping finds pathological, impulsive, and 'normal' groups among adolescents who self-harm. J Child Psychol Psychiatry 50:807-815

44. Steinhausen H-C, Haslimeier C, Winkler Metzke C (2006) The outcome of episodic versus persistent adolescent depression in young adulthood. J Affect Disord 96:49-57

45. Storey P, Hurry J, Jowitt S, Owens D, House A (2005) Supporting young people who repeatedly self-harm. J Royal Soc Promotion Health 125:71-75

46. Wild LG, Flisher AJ, Lombard C (2004) Suicidal ideation and attempts in adolescents: associations with depression and six domains of self-esteem. J Adolesc 27:611-624

47. Ystgaard M, Arensman E, Hawton K, Madge N, van Heeringen K, Hewitt A, de Wilde EJ, De Leo D, Fekete S (2009) Deliberate self-harm in adolescents: comparison between those who receive help following self-harm and those who do not. J Adolesc 32:875-891

48. Ystgaard M, Hestetun I, Loeb M, Mehlum L (2004) Is there a specific relationship between chilhood sexual and physical abuse and repeated suicidal behavior? Child Abuse Negl 28:863-876

49. Zigmond AS, Snaith RP (1983) The hospital anxiety and depression scale. Acta Psychiatr Scand 67:361-370 